\title{
Masses and spectral functions for anti-D mesons in nuclear matter and partial restoration of chiral symmetry
}

\author{
Daiki Suenaga* \\ Nagoya University \\ E-mail: Suenagadhken.phys.nagoya-u.ac.jp
}

\begin{abstract}
We investigate modifications of $\bar{D}$ mesons in nuclear matter with a partial restoration of chiral symmetry. Nuclear matter is constructed by parity doublet model and we fully respect chiral symmetry in our calculations. In the parity doublet model, we have a free parameter which is called a chiral invariant mass defined by a part of the nucleon mass invariant with respect to chiral symmetry. Then, we especially study a chiral invariant mass dependence of spectral function for $\bar{D}_{0}^{*}$ meson at normal nuclear matter density. As a result, we find that two clear peaks appear in the spectral function for $\bar{D}_{0}^{*}$ meson regarded as a $\bar{D}_{0}^{*}$ meson resonance and a threshold enhancement. The peak position of the $\bar{D}_{0}^{*}$ meson resonance moves to the lower energy regime as we increase the value of the chiral invariant mass while that of the threshold enhancement moves to the higher energy side. These changes provide us fruitful information of the value of the chiral invariant mass as well as the magnitude of partial restoration of chiral symmetry at normal nuclear matter density.
\end{abstract}

XVII International Conference on Hadron Spectroscopy and Structure - Hadron2017

25-29 September, 2017

University of Salamanca, Salamanca, Spain

* Speaker. 


\section{Introduction}

Chiral symmetry is undoubtedly spontaneously broken in the vacuum to provide a nucleon with its mass. This mechanism is broadly confirmed experimentally through the pion dynamics together with low energy theorems in association with spontaneous breakdown of chiral symmetry. However, we still have a homework that we should answer the following question: How much amount of the mass of nucleon is generated by the spontaneous breakdown of chiral symmetry? The mass of nucleon $M_{N}$ is schematically expressed as $M_{N}=m_{0}+f(\langle\bar{q} q\rangle)$, where $f(\langle\bar{q} q\rangle)$ means a part of the nucleon mass originated by the spontaneous breakdown of chiral symmetry and $m_{0}$ is called a chiral invariant mass which is invariant with respect to chiral symmetry [四]. The value of $m_{0}$ is not understood well at the present moment and the homework stated above is corresponding to determining the value of $m_{0}$. One useful way to get information of the value of $m_{0}$ is to access to density since it is expected that chiral symmetry tends to be restored at such extreme environment. Therefore, we consider nuclear matter in the present study.

In order to explore chiral symmetry and related quantities such as the value of $m_{0}$ in nuclear matter, we propose that $\bar{D}$ mesons $(\bar{D} \sim \bar{c} q)$ can be appropriate probes [《], []]. This is because $\bar{D}$ mesons contain following two advantages: First, the mass of $\bar{D}$ meson is sufficiently large in comparison with $\Lambda_{\mathrm{QCD}}$ and we can construct a concise effective Lagrangian. Second, $\bar{D}$ mesons carry only one light quark and they are belonging to a simple representation of chiral symmetry referred to as the fundamental representation. Note that we especially focus on $\bar{D}$ mesons (not $D$ mesons) in this study since $\bar{D}$ mesons do not possess an anti-light quark and we can neglect complicated pair annihilation processes.

In the present analysis, we employ a chiral partner structure for a treatment of $\bar{D}$ mesons [䧃]. In the context of the chiral partner structure, a mass difference between positive-parity meson and negative-parity meson is generated by the spontaneous breakdown of chiral symmetry. Then, we further focus on a spectral function for $\bar{D}_{0}^{*}\left(0^{+}\right)$meson [B] . Since this meson mainly decays into $\bar{D}$ $\left(0^{-}\right)$meson by emitting a pion, we expect significant changes in the spectral function for $\bar{D}_{0}^{*}$ meson in nuclear matter with the partial restoration of chiral symmetry. Our study provides information of the origin of the nucleon mass as well as the magnitude of the partial restoration of chiral symmetry at normal nuclear matter density for future experiments at FAIR and J-PARC.

This write-up is organized as follows: In Sec. 凤, we construct nuclear matter within the parity doublet model. In Sec. 及, we introduce an effective Lagrangian for $\bar{D}$ mesons based on the chiral partner structure. In Sec. 田, we show some results of the spectral function for $\bar{D}_{0}^{*}$ meson at normal nuclear matter density and give conclusions.

\section{Parity doublet model}

In this section, we give some explanations of the parity doublet model and construct nuclear matter. The nucleon is composed of three valence quarks $(N \sim q q q)$ so that it is possible to introduce following two types of nucleon fields:

$$
\begin{aligned}
& N_{1 L} \rightarrow g_{L} N_{1 L}, N_{1 R} \rightarrow g_{R} N_{1 R} \\
& N_{2 L} \rightarrow g_{R} N_{2 L}, N_{2 R} \rightarrow g_{L} N_{2 R} .
\end{aligned}
$$


In these assignments, we have defined the left-handed and right-handed nucleons $N_{1 L(2 L)}$ and $N_{1 R(2 R)}$ by eigenvalues of chirality as

$$
N_{1 L(2 L)}=\frac{1-\gamma_{5}}{2} N_{1(2)}, N_{1 R(2 R)}=\frac{1+\gamma_{5}}{2} N_{1(2)} .
$$

In Eq. (2..J), $g_{L}$ and $g_{R}$ are elements of $S U(2)_{L}$ and $S U(2)_{R}$ chiral group, respectively. The assignment for $N_{2}$ is referred to as a "mirror assignment" [四]. By taking into account parity transformation laws of $N_{1}$ and $N_{2}$ as $N_{1}(x) \rightarrow \gamma_{0} N_{1}\left(x_{p}\right)$ and $N_{2}(x) \rightarrow-\gamma_{0} N_{2}\left(x_{p}\right)\left(x_{p}=\left(x_{0},-\vec{x}\right)\right)$, we can find a Lagrangian up to first derivative terms as [[]]

$$
\begin{aligned}
\mathscr{L}_{\mathrm{PD}}= & \bar{N}_{1 R}\left(i \partial+\gamma_{0} \mu_{1}\right) N_{1 R}+\bar{N}_{1 L}\left(i \partial+\gamma_{0} \mu_{1}\right) N_{1 L}+\bar{N}_{2 R}\left(i \partial+\gamma_{0} \mu_{2}\right) N_{2 R}+\bar{N}_{2 L}\left(i \partial+\gamma_{0} \mu_{2}\right) N_{2 L} \\
& -m_{0}\left[\bar{N}_{1 L} N_{2 R}-\bar{N}_{1 R} N_{2 L}-\bar{N}_{2 L} N_{1 R}+\bar{N}_{2 R} N_{1 L}\right] \\
& -g_{1}\left[\bar{N}_{1 R} M^{\dagger} N_{1 L}+\bar{N}_{1 L} M N_{1 R}\right]-g_{2}\left[\bar{N}_{2 R} M N_{2 L}+\bar{N}_{2 L} M^{\dagger} N_{2 R}\right] \\
& -i h_{1}\left[\bar{N}_{1 L}\left(M \partial M^{\dagger}-\partial M M^{\dagger}\right) N_{1 L}+\bar{N}_{1 R}\left(M^{\dagger} \partial M-\partial M^{\dagger} M\right) N_{1 R}\right] \\
& -i h_{2}\left[\bar{N}_{2 R}\left(M \partial M^{\dagger}-\partial M M^{\dagger}\right) N_{2 R}+\bar{N}_{2 L}\left(M^{\dagger} \partial M-\partial M^{\dagger} M\right) N_{2 L}\right] \\
& +\frac{1}{4} \operatorname{tr}\left[\partial_{\mu} M \partial^{\mu} M^{\dagger}\right]+\frac{\bar{\mu}^{2}}{4} \operatorname{tr}\left[M M^{\dagger}\right]-\frac{\lambda}{16}\left(\operatorname{tr}\left[M M^{\dagger}\right]\right)^{2}+\frac{\lambda_{6}}{48}\left(\operatorname{tr}\left[M M^{\dagger}\right]\right)^{6}+\frac{1}{4} \bar{m} \varepsilon \operatorname{tr}\left[M+M^{\dagger}\right] \\
& -\frac{1}{4} \omega_{\mu v} \omega^{\mu v}+\frac{1}{2} m_{\omega}^{2} \omega_{\mu} \omega^{\mu},
\end{aligned}
$$

$M$ is a chiral field which contains $\sigma$ meson and pion as $M=\sigma+i \pi^{a} \tau^{a}\left(\tau^{a}\right.$ is the Pauli matrix and $a$ runs over $a=1,2,3)$, and this field transforms under the $S U(2)_{L} \times S U(2)_{R}$ chiral transformation as $M \rightarrow g_{L} M g_{R}^{\dagger}$. $\omega_{\mu \nu}$ is a kinetic term of $\omega$ meson defined by $\omega_{\mu \nu}=\partial_{\mu} \omega_{v}-\partial_{\nu} \omega_{\mu}$. This Lagrangian is invariant under the $S U(2)_{L} \times S U(2)_{R}$ chiral transformation except for the last term in the sixth line. Note that we have added baryon number chemical potentials $\mu_{1}$ and $\mu_{2}$ and $\omega$ meson to access to the density.

The mass matrix in Eq. (2.3]) is diagonalized by introducing following new nucleon states

$$
\left(\begin{array}{l}
N_{+} \\
N_{-}
\end{array}\right)=\left(\begin{array}{cc}
\cos \theta & \gamma_{5} \sin \theta \\
-\gamma_{5} \sin \theta & \cos \theta
\end{array}\right)\left(\begin{array}{l}
N_{1} \\
N_{2}
\end{array}\right)
$$

with the mixing angle $\theta$ satisfying

$$
\tan 2 \theta=\frac{2 m_{0}}{\left(g_{1}+g_{2}\right) \sigma_{0}} .
$$

In Eqs. ([2.4) and ([2.5), we have described the spontaneous breakdown of chiral symmetry by a mean field of $\sigma$ meson $\left(\sigma_{0}\right)$. By using the parity transformation laws for $N_{1}$ and $N_{2}$, we can notice that $N_{+}$is a positive-parity nucleon while $N_{-}$is a negative-parity nucleon. Therefore, we regard $N_{+}$ as the nucleon and $N_{-}$as the $N^{*}(1535)$. After diagonalizing the mass matrix, we find the masses of the nucleon $\left(m_{+}\right)$and $N^{*}(1535)\left(m_{-}\right)$read

$$
m_{ \pm} \equiv \frac{1}{2}\left[\sqrt{\left(g_{1}+g_{2}\right)^{2} \sigma_{0}^{2}+4 m_{0}^{2}} \mp\left(g_{2}-g_{1}\right) \sigma_{0}\right] .
$$




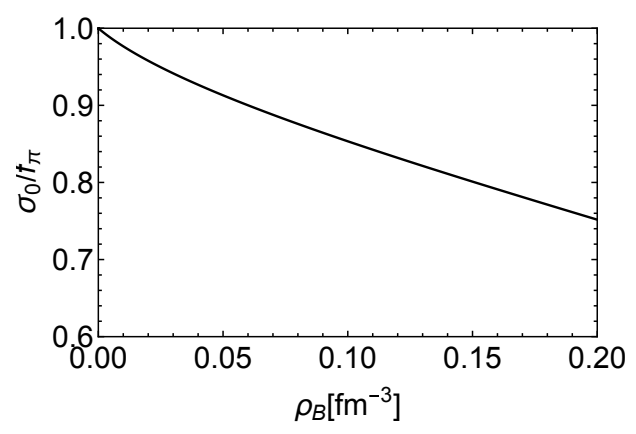

Figure 1: A density dependence of the mean field of $\sigma$ meson $\left(\sigma_{0}\right)$ with $m_{0}=500 \mathrm{MeV}$.

These mass formulae clearly show $m_{0}$ is the chiral invariant mass since this term survives at which a restoration of chiral symmetry indicated by $\sigma_{0} \rightarrow 0$ occurs.

In our study, nuclear matter is described by one-loops with respect to the nucleon and $N^{*}(1535)$. To fix the parameters, we adopt the nucleon mass $m_{N}=939 \mathrm{MeV}, N^{*}(1535)$ mass $m_{N^{*}}=1535$ $\mathrm{MeV}$, the pion decay constant $f_{\pi}=93 \mathrm{MeV}$, pion mass $m_{\pi}=140 \mathrm{MeV}, \omega$ meson mass $m_{\omega}=783$ $\mathrm{MeV}$, the decay width $\Gamma_{N^{*} \rightarrow N \pi}=75 \mathrm{MeV}$ and the nucleon axial charge $g_{A}=1.27$ as inputs. We also use the saturation density $\rho_{B}^{*}=0.16 \mathrm{fm}^{-3}$, the incompressibility $K=270 \mathrm{MeV}$ and the bounding energy $E_{B}=16 \mathrm{MeV}$ as input parameters. Note that by using these inputs, only the chiral invariant mass $m_{0}$ remains as a free parameter in our model.

A density dependence of the mean field of $\sigma$ meson $\left(\sigma_{0}\right)$ is determined by solving a gap equation for $\sigma_{0}$, and the resultant density dependence of $\sigma_{0}$ is shown in Fig. $\square$ with $m_{0}=500 \mathrm{MeV}$ as an example. This figure manifests the partial restoration of chiral symmetry in nuclear matter. The magnitude of restoration at the normal nuclear matter density $\rho_{B} \approx 0.16 \mathrm{fm}^{-3}$ is $\sigma_{0} / f_{\pi} \approx 0.8$.

\section{Lagrangian for $\bar{D}$ mesons}

In this section, we construct an effective Lagrangian for $\bar{D}$ mesons by the chiral partner structure. Heavy-light meson fields such as $\bar{D}$ mesons carry an anti-heavy quark and a light quark. Then we can introduce left-handed and right-handed heavy-light meson fields $H_{L}$ and $H_{R}$ which transform under the chiral transformation as

$$
H_{L} \rightarrow g_{L} H_{L}, H_{R} \rightarrow g_{R} H_{R} .
$$

Note that these fields are schematically depicted as $H_{L} \sim q_{L} \bar{Q}$ and $H_{R} \sim q_{R} \bar{Q} . H_{L}$ and $H_{R}$ include spin-0 and spin-1 heavy-light mesons collectively thanks to the Heavy Quark Spin Symmetry (HQSS), and in terms of the HQSS, they transform as

$$
H_{L} \rightarrow H_{L} S^{\dagger}, H_{R} \rightarrow H_{R} S^{\dagger} .
$$

Therefore, we can find a Lagrangian for $\bar{D}$ mesons interacting with light mesons as

$$
\begin{aligned}
\mathscr{L}_{\mathrm{HMET}}= & \operatorname{tr}\left[\bar{H}_{L}(i v \cdot \partial) H_{L}\right]+\operatorname{tr}\left[\bar{H}_{R}(i v \cdot \partial) H_{R}\right]+\frac{\Delta_{m}}{2 f_{\pi}} \operatorname{tr}\left[\bar{H}_{L} M H_{R}+\bar{H}_{R} M^{\dagger} H_{L}\right] \\
& +i \frac{g}{2 f_{\pi}} \operatorname{tr}\left[\bar{H}_{R} \gamma_{5} \gamma^{\mu} \partial_{\mu} M^{\dagger} H_{L}-\bar{H}_{L} \gamma_{5} \gamma^{\mu} \partial_{\mu} M H_{R}\right]+\cdots
\end{aligned}
$$


where $v^{\mu}$ is the velocity of heavy-light mesons. $H_{L}$ and $H_{R}$ are convenient for constructing the Lagrangian since the chiral representation is transparent. Parity eigenstates of heavy-light mesons are converted via following relations:

$$
H_{L}=\frac{1}{\sqrt{2}}\left(G+i \gamma_{5} H\right), H_{R}=\frac{1}{\sqrt{2}}\left(G-i \gamma_{5} H\right) .
$$

$G$ and $H$ doublets include $G=\left\{\bar{D}_{0}^{*}\left(0^{+}\right), \bar{D}_{1}\left(1^{+}\right)\right\}$and $H=\left\{\bar{D}\left(0^{-}\right), \bar{D}^{*}\left(1^{-}\right)\right\}$, respectively, which can be parametrized as

$$
G=\left[\bar{D}_{0, v}^{*}-i \gamma_{5} \bar{D}_{1, v}\right] \frac{1+\vartheta}{2}, H=\left[\bar{D}_{v}^{*}+i \gamma_{5} \bar{D}_{v}\right] \frac{1+\vartheta}{2} .
$$

Substituting Eq. (B.4) into the Lagrangian in Eq. (B.3) together with Eq. (B.5)), we can obtain a Lagrangian for $\bar{D}$ mesons. It is more convenient to rewrite the Lagrangian into a relativistic form by defining relativistic fields $\bar{D}$ via $\bar{D}=\frac{1}{\sqrt{m}} \mathrm{e}^{-i m v \cdot x} \bar{D}_{v}$.

After employing the procedure stated above, we find mass formulas for $\bar{D}$ mesons as

$$
m_{\left(\bar{D}_{0}^{*}, \bar{D}_{1}\right)}^{*}=m+\frac{\Delta_{m} \sigma_{0}}{2 f_{\pi}}, m_{\left(\bar{D}, \bar{D}^{*}\right)}^{*}=m-\frac{\Delta_{m} \sigma_{0}}{2 f_{\pi}} .
$$

In these formulae, masses of $\bar{D}$ and $\bar{D}^{*}$ meson coincide while those $\bar{D}_{0}^{*}$ and $\bar{D}_{1}$ meson coincide which manifestly shows a consequence of the HQSS. Eq. (B.6) tells us that the mass difference between $m_{\left(\bar{D}_{0}^{*}, \bar{D}_{1}\right)}$ and $m_{\left(\bar{D}, \bar{D}^{*}\right)}^{*}$ reads

$$
\Delta_{m}^{*}=\equiv m_{\left(\bar{D}_{0}^{*}, \bar{D}_{1}\right)}-m_{\left(\bar{D}, \bar{D}^{*}\right)}^{*}=\frac{\Delta_{m}}{f_{\pi}} \sigma_{0} .
$$

This equation claims that the mass difference between positive-parity $\bar{D}$ meson and negative-parity $\bar{D}$ meson is generated by the spontaneous breakdown of chiral symmetry, and this feature is referred to as the chiral partner structure [四].

The parameters $m, \Delta_{m}$ and $g$ are fixed by the spin-averaged mass of $H$ doublet, the spinaveraged mass of $G$ doublet and the decay width of $\Gamma_{D^{*} \rightarrow D \pi}$ in the vacuum.

\section{Results and Conclusions}

In this section, we show the main results on the spectral function for $\bar{D}_{0}^{*}$ meson at the normal nuclear matter density and give conclusions of our study. Nuclear matter is constructed by the parity doublet model derived in Sec. $\square$ and $\bar{D}$ mesons are introduced by the chiral partner structure described in Sec. [1.

In Sec. [1], the Lagrangian for $\bar{D}$ mesons in a heavy quark limit is derived. In this section, we introduce a small violation of the HQSS to make our arguments more realistic. In order to calculate the spectral function for $\bar{D}_{0}^{*}$ meson in nuclear matter, we need to get a self-energy of $\bar{D}_{0}^{*}$ in medium. The self-energy includes Hartree-type and Fock-type one-loop corrections as well as the mean field $\sigma_{0}$. In the present analysis, we evaluate the Fock-type one-loop correction by a $\bar{D} \pi$ one-loop alone. In the vacuum. $\bar{D}_{0}^{*}$ decays into $\bar{D}$ and pion mainly, and we expect that the self-energy of $\bar{D}_{0}^{*}$ meson is dominated by the $\bar{D} \pi$ one-loop. We confirm that the other one-loop corrections contribute 


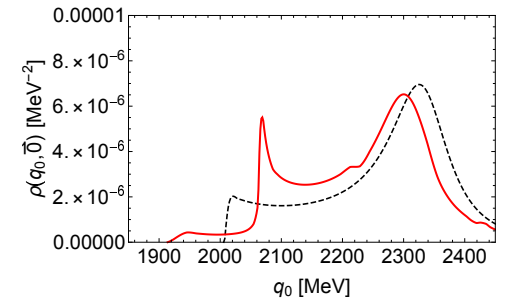

(a) $m_{0}=500 \mathrm{MeV}$.

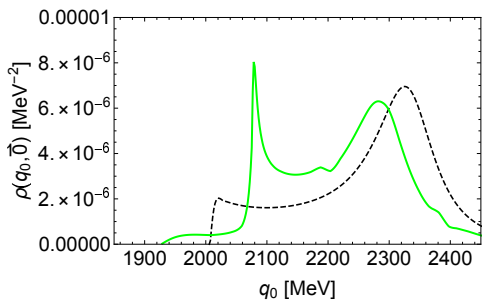

(b) $m_{0}=700 \mathrm{MeV}$.

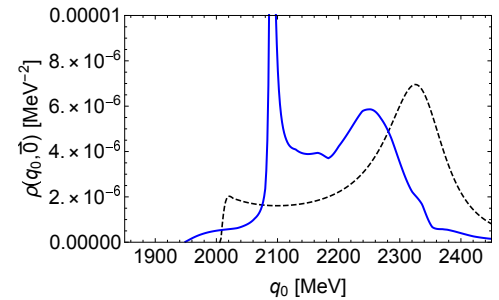

(c) $m_{0}=900 \mathrm{MeV}$.

Figure 2: (color online) Spectral functions for $\bar{D}_{0}^{*}$ meson at $\rho_{B}=0.16 \mathrm{fm}^{-3}$ and $\vec{q}=\overrightarrow{0}$ with (a) $m_{0}=500$ $\mathrm{MeV}$, (b) $m_{0}=700 \mathrm{MeV}$, (c) $m_{0}=900 \mathrm{MeV}$. The colored curves are the results, and dashed black curve is the spectral function in the vacuum.

negligibly in fact. Note that we fully respect chiral symmetry the parity doublet model possesses in calculating the one-loop corrections.

The resulting spectral function for $\bar{D}_{0}^{*}$ is shown in Fig. $\square$ at $\rho_{B}=0.16 \mathrm{fm}^{-3}$ with several values of $m_{0}$. We take $\vec{q}=\overrightarrow{0}$ in our calculation. In this figure, we can find two clear peaks. The right peak is a resonance state of $\bar{D}_{0}^{*}$ meson, and its peak position shifts to the lower energy regime as we increase the value of $m_{0}$. The left peak is a threshold enhancement which is caused by a virtual state or a bound state of $\bar{D} \pi$ state, and this peak shifts to the higher energy as the increase of $m_{0}$. These characteristic behaviors show that the partial restoration of chiral symmetry is accelerated by increasing the value of $m_{0}$.

These characteristic structures of the spectral function for $\bar{D}_{0}^{*}$ meson provide us with fruitful information of the value of the chiral invariant mass $m_{0}$ in addition to the magnitude of partial restoration of chiral symmetry at normal nuclear matter density. Especially, the threshold enhancement has a sharp peak and can remarkable enhance. Therefore, the threshold enhancement is a proper probe to investigate them. The shape of spectral function for $\bar{D}_{0}^{*}$ meson in nuclear matter appears in a double differential cross section of " $\bar{p}+A \rightarrow\left(\bar{D}_{0}^{*}\right.$ in medium $)+D$ " for instance. Then, it is interesting to evaluate the double differential cross section to provide observables for the future experiments at FAIR or J-PARC.

\section{References}

[1] C. E. DeTar and T. Kunihiro, Phys. Rev. D 39, 2805 (1989), D. Jido, M. Oka and A. Hosaka, Prog. Theor. Phys. 106, 873 (2001).

[2] D. Suenaga and M. Harada, Phys. Rev. D 93, no. 7, 076005 (2016) doi:10.1103/PhysRevD.93.076005, M. Harada, Y. L. Ma, D. Suenaga and Y. Takeda, arXiv:1612.03496 [hep-ph].

[3] D. Suenaga, S. Yasui and M. Harada, Phys. Rev. C 96, no. 1, 015204 (2017) doi:10.1103/PhysRevC.96.015204.

[4] M. A. Nowak, M. Rho and I. Zahed, Phys. Rev. D 48, 4370 (1993) doi:10.1103/PhysRevD.48.4370, W. A. Bardeen and C. T. Hill, Phys. Rev. D 49, 409 (1994).

[5] Y. Motohiro, Y. Kim and M. Harada, Phys. Rev. C 92, no. 2, 025201 (2015) Erratum: [Phys. Rev. C 95, no. 5, 059903 (2017)] doi:10.1103/PhysRevC.92.025201, 10.1103/PhysRevC.95.059903, D. Suenaga, arXiv:1704.03630 [nucl-th]. 